\title{
Stability Control Structure of Hovercraft Prototype Utilising PID Controller
}

\author{
Bhaskara Rao Yenugula* ${ }^{* 1}$, Md. Zia-ur-Rahman ${ }^{2}$ \\ ${ }^{1}$ Department of Electrical Engineering(EEEM), AMET University, Chennai, India \\ ${ }^{2}$ Department of Electronics \& Communication Engineering, KL University, Guntur, India \\ ${ }^{*}$ Corresponding author, e-mail: eei@journal.uad.ac.id
}

\begin{abstract}
Hovercraft is a method of transportation as an option for clients who remain on the waterway and swamp surface. The issue with hovercraft is when dubious climate and natural condition, e.g. wind speed and wave tallness exasperate solidness of hovercraft to jeopardise the driver. We propose an approach to keep up adjust of the hovercraft by controlling the focal point of gravity (PG) to be determined position. The controller monitors the position of load to change the position. A 6-DOF IMU Sensor MPU 6050 was utilised to create information as an examination with setpoint. PID control strategy was employed. The test outcome demonstrates that the model of air cushion vehicle could keep its adjust the axis orientation of the roll in spite of the fact that it was less compelling in the pitch pivot direction.
\end{abstract}

Keywords: stability control structure, hovercraft prototype, PID Controller, Point of Gravity (PG), setpoint

\section{Introduction}

Indonesia is an archipelago nation with over $70 \%$ of its zone comprising of waters and inexhaustible stream ways [1]. Other than that, Indonesia has significant hazard from cataclysmic events, for example, tremor, surge, and even torrent. Because of that geographic condition, Indonesia needs a vehicle which can move quick and stable in many conditions, to use in transportation, crisis, drift protect, and inquiry and save [2]. Since the air cushion vehicle can move in off-road conditions, this vehicle is one of the arrangements which can fulfil those prerequisites [3]. A hovercraft, or Air-Cushion Vehicle, is a land and water capable vehicle intended to go over any adequately smooth surface-land or water bolstered by a pad of gradually moving, little weight air, catapulted downwards against the surface close underneath it [4]. To create the air pad, the propeller is utilised to give the lift by keeping a low-weight. One inadequacy of this air cushion vehicle is its restricted moving [5]. At the season of a move, development will influence the introduction point of hovercraft, because of changes in the incline on the turning pivot in hovercraft body [6]. This hovercraft introduction point change may bring about the loss of steadiness in hovercraft body. Along these lines, control of hovercraft body is necessary to keep up in the stable position so that the slant of hovercraft can settle and adjusted [7].

One approach to making the hovercraft body stays stable is to control the focal point of gravity of the air cushion vehicle. Translational pivot X, Y, Z and turn edges of pitch, roll and yaw are utilised as the reference to control the focal point of gravity of the hovercraft [8]. What's more, the severity and speeding up are essential information as a correlation for corner setpoint. The 6-pivot MPU-6050 module is a blend of accelerometer and Gyroscope sensors that can utilise this reason. Likewise, among numerous strategies, PID and fuzzy techniques have been employed to give the information setpoint and keep up hovercraft body tilt on the coveted setpoint. In this paper described that the wind power generation [9-11].

In this examination, we propose a plan of control framework on the model of air cushion vehicle with the reason to keep up the air cushion vehicle body position in the stable state while moving. The PID controller was chosen because of its straightforwardness yet proficient, as it can customise with less complicated microcontroller-based hardware. 


\section{Research Method}

Mechanical frameworks in the model of air cushion vehicle are exceptionally compelling for dependability when on the development. In particular, the outline of air cushion vehicle model must have the COG (Center of Gravity) moving toward the introduction tomahawks so that the development in keeping up body dependability should be possible with less bother. Figure 1 demonstrates the outline point of view of the model.

With reference, the position and weight of every segment, the focal point of gravity (COG) can figure. The COG computation required because with a specific end goal to get static strength, the heap must be disseminated equally over the surface territory of the air cushion vehicle model. To meet that necessity, the COG must set close to the focal point of the type body, hence makes it simple to make development in any edge or heading. The symmetry amongst left and right half of body along the inside line is critical in a key position of the framework while pushing ahead. Also, the reference point $(0,0)$ chosen at the out and downright point.

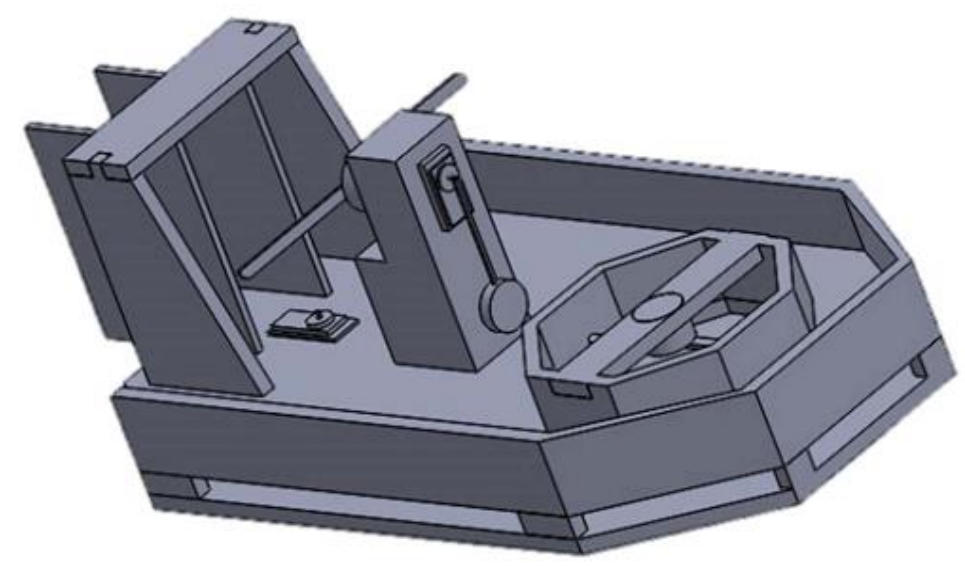

Figure 1. Hovercraft prototype design

\section{Results and Analysis}

Segments with their particular weight appropriate so the heaviness of the considerable number of parts can be focused amidst prototype at coordinate $(12.5,22.5)$ to encourage the plan of control framework on an air cushion vehicle model's adjust. The reference point is the beginning stage of deciding the separation of every segment.

After the parts placed inopportune place, then the subsequent step is to compute the coordinates of the focal point of gravity. It is important that every segment accepted as direct mass body toward streamlining the figuring of the central point of gravity. Factors $X$ and $Y$ are the relative positions of a reference point. The weight computes from $\mathrm{W}=\mathrm{m} . \mathrm{g}$, with mas mass and gravity $\mathrm{g}=9.8 \mathrm{~ms}^{-2}$.

In the model, the balance is controlled utilising a PID controller. The mistake of the set point is produced from the sensor information and after that computed into PID. By and large, the 6 DOF IMU sensor provides roll and pitch edges. This side is contrasted and the information set point. Angle mistake is sustained into PID controller input. The mistake is then ascertained to create the PWM signalyield.

As the essential piece of the framework, 6 DOF MPU-6050 is a blend of two sorts of sensors: accelerometer and gyroscope sensor made by InvenSense with I2C correspondence interface. The MPU-6050 consolidates accelerometer and gyroscope in a single board. These sensors can identify increasing speed in three $\operatorname{axes}(\mathrm{x}, \mathrm{y}$, and $\mathrm{z})$ and the precise speed in three axes $(x, y$, and $z)$.

The two information sources bring noise, each of which is high and low-frequency noise. Along these lines, two sorts of channels utilised. The signal with high-frequency noise was separated by the lowpass channel, while the info containing low-frequency noise sifted with 
high pass chains. The yield recreation of both signals after channels produces motion without clamour already connected with a sensor.

\section{Conclusion}

The air cushion vehicle model has been created with the PID controller to keep up its steadiness. The heap adjust instrument was utilised with the worry on the focal point of gravity keeping in mind the end goal to remunerate any change of pitch and move edge. The testing comes about uncovered that the air cushion vehicle could push ahead over the ground effortlessly, and within sight of unsettling influences, it could recoup to its steady position. Angel mistakes were found for around $0.51^{\circ}$ and $1.11^{\circ}$ in roll and pitch points, separately. The standard time to achieve strength was 16.96 seconds.

\section{References}

[1] Fantoni I, Lozano R, Mazenc F, Pettersen KY. Stabilization of a Nonlinear Underactuated Hovercraft. In Decision and Control, 1999. Proceedings of the 38th IEEE Conference. 1999; 3: 2533-2538.

[2] Othmane Boughazi, Abdelmadjid Boumedienne, Hachemi Glaoui. Sliding Mode Backstepping Control of Induction Motor. International Journal of Power Electronics and Drive Systems (IJPEDS). December 2014; 4(4): 481-488.

[3] Wang CC, Su JP. Fuzzy Gain Scheduled Integral Control and its Application to a Hovercraft Vessel with Uncertainties. In Innovative Computing, Information and Control, 2006. ICICIC'06. First International Conference. 2006; 1: 501-504.

[4] Rekha Chaudhary, Arun Kumar Singh, Saligram Agrawal. A Novel Approach to PID Controller Design for Improvement of Transient Stability and Voltage Regulation of Nonlinear Power System. International Journal of Electrical and Computer Engineering (IJECS). October 2016; 6(5): 22252238.

[5] Shi X, Liu Z, Fu M, Wang C. Amphibious Hovercraft Course Control Based on Support Vector Machines Adaptive PID. In Automation and Logistics (ICAL). 2011. IEEE International Conference on 2011: 287-292.

[6] Tanaka K, Iwasaki M, Wang HO. Stable Switching Fuzzy Control and its Application to a Hovercraft Type Vehicle. In Fuzzy Systems, 2000. FUZZ IEEE 2000. The Ninth IEEE International Conference on 2000; 2: 804-809.

[7] Okafor BE. Development of a Hovercraft Prototype. International Journal of Engineering and Technology. 2013: 3(3).

[8] Luo Z, Yu F. Load Distribution Control System Design for a Semi-track air-cushion Vehicle. Journal of Terramechanics. 2007; 44(4): 319-325.

[9] Patel AKP, Sivaraman A. The Past, Present and Future of the Offshore Wind Power Technology-A Review. In Offshore Technology Conference. Offshore Technology Conference, 2016.

[10] Mohamed Najeh Lakhoua, Naoueli Walid, Chakroun Atef. System Analysis of a Hybrid Renewable Energy System. International Journal of Electrical and Computer Engineering (IJECE). June 2014; 4(3): 343-350.

[11] Gopinath, S. Navigational \& Safety Assessment of Wind Farm Support Vessels, RINA. Royal Institution of Naval Architects-Design and Operation of Wind Farm Support Vessels. 2015: 43-46. 